\title{
Mejores prácticas para diseñar mezclas asfálticas con pavimento asfáltico recuperado (RAP)
}

\section{Recommended best practices for using rap in asphalt pavements for Costa Rica}

\section{Ing. Fabricio Leiva-Villacorta, Ph.D., MBA}

NCAT, Universidad de Auburn, Alabama

leivafa@auburn.edu
Ing. Adriana Vargas-Nordcbeck, Ph.D., MBA

NCAT, Universidad de Auburn, Alabama

vargaad@auburn.edu

Fecha de recepción: 21 de febrero de 2017 / Fecha de aprobación: 26 de julio de 2017

\section{RESUMEN}

La utilización de pavimento asfáltico recuperado (RAP en inglés) en carreteras se ha convertido en una estrategia importante para ayudar a compensar el aumento del precio de las materias primas y mejorar la sostenibilidad de la infraestructura de transporte. La forma de caracterizar los materiales de reciclado, el diseño de mezcla, los ensayos de desempeño y las prácticas constructivas no siguen ninguna práctica estándar conocida. El objetivo de este estudio fue el identificar las mejores prácticas para el diseño y producción de mezclas asfálticas con RAP. El documento propuesto proporciona una guía para el uso eficaz de los materiales de RAP e incluye recomendaciones sobre la manipulación y el almacenamiento del RAP, ensayos de este material, producción de mezclas que contienen RAP, y las prácticas de control de calidad durante la producción de las mezclas que contienen RAP.

PALABRAS CLAVES: RAP, mezcla asfáltica, desempeño, pavimentos, mejores practicas.

\begin{abstract}
Utilization of RAP in asphalt pavements has become an important strategy to help offset rising raw material prices and to improve the sustainability of our transportation infrastructure. Characteristics of the recycled materials, mix designs, performance and construction practices do not follow any known standard practice. The objective of the proposed document is to identify best practices for specification, design, production, testing, and placement of RAP mixes. The proposed document provides guidance for the effective use of reclaimed asphalt pavement (RAP) materials in pavements specifically for Costa Rica. The document includes recommendations on when milling should be considered as part of pavement rehabilitation, best practices for handling and stockpiling RAP, testing the RAP, designing mixes with RAP, production of mixes containing RAP, and quality control practices during production of mixtures containing $R A P$.
\end{abstract}

KEYWORDS: RAP, asphalt mixture, performance, pavements, best practices. 


\section{INTRODUCCIÓN}

Los materiales asfálticos recuperados durante las operaciones de rehabilitación o reconstrucción pueden ser utilizados nuevamente en mezclas asfálticas como agregado y ligante complementario. El aumento del uso de RAP en proyectos de construcción de carreteras y la rehabilitación en todo el mundo se ha convertido en una estrategia importante para ayudar a compensar los crecientes costos de las materias primas y mejorar la sostenibilidad de las infraestructuras de transporte. El uso de RAP reduce los costos asociados a los materiales y el transporte. Los costos de los materiales comprenden aproximadamente 70 por ciento del costo total de producción de una mezcla asfáltica, siendo en este caso, el ligante asfáltico el material de mayor costo (Copeland, 2011).

Mediante la sustitución de una parte de los materiales vírgenes con RAP, especialmente en las capas intermedia y superficial, se puede obtener mayores beneficios económicos. En estas capas se utiliza el ligante (material más costoso) para proporcionar una resistencia a la tracción, proteger ante el daño por humedad y proporcionar una superficie de rodadura regular y resistente al deslizamiento.

Khandal y Mallik (1997) estiman que un ahorro de hasta un 34 \% podría ser generado para mezclas que contienen hasta un $50 \%$ de RAP. McDaniel y Nantung (2005) informaron en un análisis beneficio-costo, realizado por el Departamento de Transportes de Indiana, que el ahorro en materiales puede ir desde \$330,000 USD al año, cuando se incorpora $5 \%$ de RAP a un poco mas de 5 millones de toneladas de material de base.

El uso de RAP también conserva la energía, preserva los recursos naturales y reduce la cantidad de escombros de construcción que se coloca en los vertederos. Un estudio preparado para el Departamento de Transporte del Estado de Nueva York (National Technology Development LLC, 2009) cuantifica la energía y el impacto ambiental del uso de RAP en mezclas asfálticas a través de varios modelos matemáticos que combinan el secadocalefacción, transporte, procesamiento y energías caloríficas. Se encontró que el uso de RAP dio lugar a ahorros de energía significativos, y que la diferencia en el consumo de energía se ve afectada por el contenido de RAP, la humedad en el RAP, y la temperatura de descarga de la mezcla. Además, se estima que el uso de bajos contenidos de RAP tiende a aumentar las emisiones de $\mathrm{CO}_{2}$ mientras que lo contrario es cierto para un alto contenido de RAP. Sin embargo, la reducción de las emisiones de $\mathrm{CO}_{2}$ del uso de RAP se presenta principalmente por la distancia más corta o nula de acarreo del RAP.
Está claro que la rehabilitación efectiva de pavimentos asfálticos a veces requiere la eliminación de capas asfálticas envejecidas. $\mathrm{Si}$ las agencias, departamentos de transporte y la industria no han desarrollado técnicas adecuadas para utilizar RAP, los costos de rehabilitación de pavimentos asfálticos aumentarían significativamente para cubrir los costos de eliminación de los materiales no reciclados. Por lo tanto, el uso eficaz de RAP resuelve un problema ambiental más amplio en que el material no ocupa espacio en los vertederos.

Este documento proporciona una guía para el uso eficaz de los materiales asfálticos recuperados. El documento incluye recomendaciones sobre las mejores prácticas para la manipulación, el almacenamiento del RAP, el diseño de mezcla con RAP, ensayos de laboratorio, producción de mezclas que contienen RAP y las prácticas de control de calidad durante la producción.

\section{OBJETIVO}

El objetivo de este estudio fue el identificar las mejores prácticas para el diseño y producción de mezclas asfálticas con RAP. El objetivo secundario es sugerir revisiones y ajustes a especificaciones, a las prácticas de preservación del pavimento y elaborar directrices para el uso de los materiales recuperados en pavimentos asfálticos.

\section{PRÁCTICAS PROPUESTAS}

Este documento se divide en las siguientes áreas: Manejo del material recuperado, caracterización del RAP, diseño de la mezcla asfáltico, producción y control de calidad de las mezclas que contienen RAP. Dentro de algunas de las secciones se proponen especificaciones.

\section{Manejo del material recuperado}

La variabilidad del RAP hace que sea difícil controlar el contenido de asfalto, la granulometría y los vacíos de aire de la mezcla durante la producción, especialmente en porcentajes altos de RAP (Solaimanian y Tahmoressi, 1996). El uso de técnicas adecuadas para el almacenamiento y procesamiento del RAP puede ayudar a controlar su variabilidad. Estas técnicas incluyen la eliminación de la contaminación, la separación de los apilamientos de RAP de diferentes fuentes, el procesamiento (trituración o fraccionamiento) del RAP, el almacenando del RAP procesado utilizando una superficie pavimentada, inclinada para reducir el contenido de humedad, y finalmente, la caracterización del RAP inmediatamente después de ser procesado (Zhou et al. 2010). 
Reducir al mínimo el contenido de humedad en los apilamientos de RAP es importante para la calidad de la mezcla y la eficiencia de la planta. Si el material de RAP tiene un alto contenido de humedad, esta primero se debe convertir en vapor antes de que el ligante de asfalto puede ser calentado lo suficiente como para ser reactivado. Esta energía que se consume en el secado del RAP afecta a la temperatura a la que el agregado virgen se calienta. Para reducir al mínimo la humedad en el RAP, los apilamientos deben ser construidos en forma cónica en áreas que drenan el agua lejos de la base. Otras medidas, tales como cubrir los apilamientos de RAP con un techo de un edificio abierto por los lados también puede ayudar a minimizar el contenido de humedad (Copeland, 2011).

El procesamiento del RAP implica uno o más pasos para crear materiales consistentes que pueden ser utilizados en un alto porcentaje y cumplir con los estándares de las mezclas de asfalto de alta calidad (Copeland, 2011). El procesamiento adicional de material triturado puede o no ser necesario para obtener una consistencia adecuada. Como mínimo, los materiales de RAP deben ser tamizados a través de una malla de $38.1 \mathrm{~mm}$ antes de entrar en la planta para eliminar los trozos más grandes de RAP ya que el calor no puede penetrar a fondo a través de densas partículas de RAP mayor que $38.1 \mathrm{~mm}$ durante el proceso de mezcla. Se recomienda establecer tolerancias en la granulometría del RAP antes y después del procesamiento de $\pm 4 \%$ del material pasando el tamiz No 8 y $\pm 1 \%$ en el tamiz No. 200 (West et al. 2013). La mayoría de las fuentes de RAP se volverán un agregado grueso bien graduado, comparable a, o tal vez ligeramente más fino y más variable que, agregados naturales triturados (Chesner et al. 1998).

Se deben hacer pruebas de laboratorio para determinar si el contenido de asfalto y la granulometría son consistentes con los resultados utilizados en el diseño de la mezcla y que el material es constante a través de la reserva de RAP. El contenido de asfalto y las pruebas de granulometría de los materiales utilizados en la producción de mezcla deben ser obtenidos a una frecuencia de una prueba por cada 1000 toneladas de material utilizado (West et al. 2013).

Se considera una buena práctica incluir al menos 10 resultados para estimar las estadísticas del apilamiento. La desviación estándar máxima recomendada para el contenido de asfalto es de $0.5 \%$. La desviación estándar máxima recomendada para el porcentaje de material pasando el tamiz de la mediana es de $5.0 \%$ y la desviación estándar máxima recomendada para el porcentaje pasando el tamiz de $0.075 \mathrm{~mm}$ es del $1.5 \%$ (West et al. 2013).

\section{Métodos de ensayo para la caracterización del RAP}

Se recomienda el método de extracción de disolvente (AASHTO $\mathrm{T}$ 164) para la determinación del contenido de asfalto de muestras de RAP. El ligante asfáltico recuperado debe ser analizado para determinar su grado de desempeño (PG) utilizando AASHTO M 320. La granulometría del agregado extraído de las muestras de RAP debe ser determinado de acuerdo con AASHTO T 30. La gravedad específica $\left(\mathrm{G}_{\mathrm{sb}}\right)$ debe ser determinada usando AASHTO $\mathrm{T} 84$ y $\mathrm{T} 85$ para las porciones finas y gruesas, respectivamente, del agregado recuperado de los ensayos de extracción. No se recomienda la estimación de la gravedad específica del agregado del RAP a partir de la gravedad específica máxima teórica del $\operatorname{RAP}\left(\mathrm{G}_{\mathrm{mm}}\right)$ ya que esto dará lugar a un $\mathrm{G}_{\mathrm{sb}}$ sobreestimado y un error en el VMA calculado que probablemente daría lugar a una mezcla de pobre desempeño.

\section{Diseño de mezcla asfáltica con RAP}

La metodología de diseño de mezcla Superpave para las mezclas que incorporan RAP es similar al diseño de la mezcla de todos los materiales vírgenes, con las siguientes excepciones (National Technology Development LLC, 2009):

- El agregado del RAP es tratado como otro de los apilamientos para mezclar y pesar, pero se debe calentar a temperaturas inferiores para evitar cambiar las propiedades del ligante del RAP;

- La gravedad específica del agregado de RAP debe ser determinado;

- La cantidad de ligante aportado por el RAP debe tenerse en cuenta cuando se dosifican los agregados;

- El contenido total de asfalto de la mezcla incluye el ligante proporcionado por el RAP; y

- Un cambio en el grado del ligante virgen puede ser necesaria dependiendo de la cantidad de RAP, del grado de desempeño deseado, y la rigidez del ligante del RAP. Para las mezclas que contienen alta cantidad de RAP, este proceso implica el uso de un gráfico de mezcla o la ecuación de mezcla para determinar la cantidad de RAP a utilizar, si el grado de desempeño del ligante virgen es conocido o para seleccionar el grado del ligante virgen si se conoce el porcentaje de ligante del RAP. 


\section{Selección del Grado de Desempeño del Ligante Virgen}

Hay varios factores que pueden afectar el grado de mezcla para cualquier diseño de mezcla en particular, incluida la compatibilidad del RAP y ligantes vírgenes, temperatura de mezclado, tiempo de mezclado, rigidez/viscosidad del ligante del RAP y ligantes vírgenes y las proporciones relativas de la RAP y ligantes vírgenes. La norma AASHTO R 35 recomienda tres niveles de uso de RAP. El primer nivel establece la cantidad máxima de RAP (15\%) que se puede utilizar sin cambiar el grado del ligante virgen. El segundo nivel, de 15 a $25 \%$, se puede utilizar cuando el grado de desempeño del ligante virgen se reduce en un grado. El tercer nivel es para los contenidos de RAP superiores (más del $25 \%$ ); para estos contenidos más altos, es necesario extraer, recuperar, y ensayar el ligante RAP para construir un gráfico de mezcla.

La Figura 1 muestra un ejemplo del proceso de selección de contenido de RAP. Para este ejemplo, se requiere un ligante PG

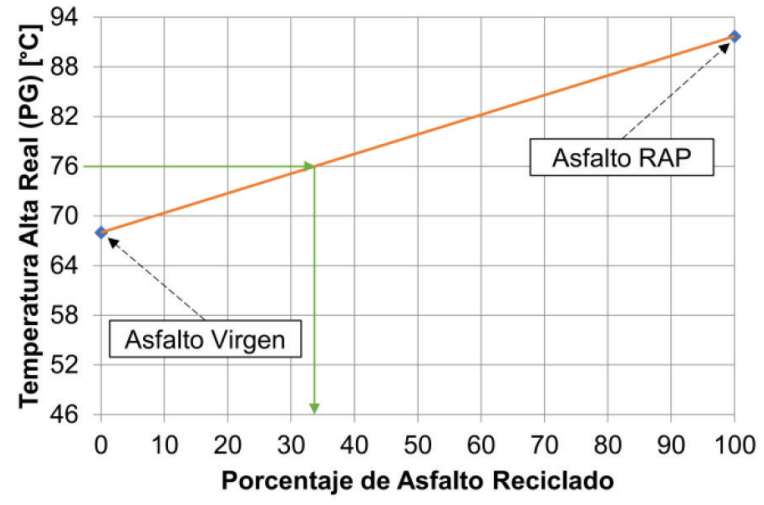

76-22 para un proyecto. Al utilizar las temperaturas críticas reales que definen el grado de desempeño del ligante virgen y el extraído del RAP se observa que un contenido de $34 \%$ de RAP proveerá el grado PG especificado.

\section{Ensayos para evaluar el desempeño del ligante asfáltico}

El contenido de ligante en el RAP varía típicamente entre 3 y 7 por ciento en peso. Este ligante es más rígido debido al envejecimiento causado por la exposición del pavimento al oxígeno atmosférico (oxidación) durante el uso y la intemperie (Chesner et al. 1998). Por lo tanto, es importante evaluar la mezcla resultante de ligantes (virgen/RAP) para determinar la forma en que la porción del ligante envejecido puede afectar al desempeño. Existen varias pruebas que han sido utilizadas como métodos para evaluar el desempeño ligante asfáltico en Costa Rica. La mayoría de estas pruebas requieren un mayor desarrollo, estudios de aceptación y precisión, y la investigación de validación de campo antes de que sean adecuados para su uso en las especificaciones.

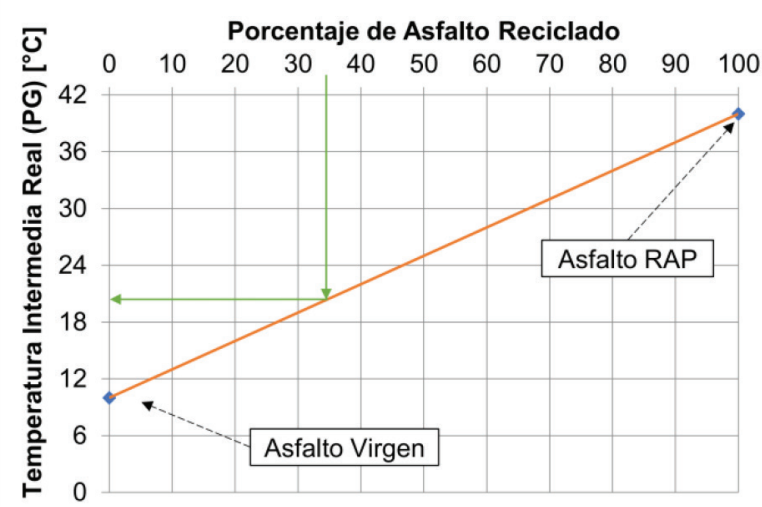

Figura 1. Ejemplo de escogencia del porcentaje de RAP por la mezcla de ligantes

\section{Ensayo de agrietamiento por fatiga}

Para caracterizar el comportamiento a la fatiga del ligante, un análisis de fatiga con carga cíclica se puede aplicar utilizando el Reómetro Dinámico de Cortante. El ensayo se realiza a la temperatura intermedia del grado PG y consiste en someter la muestra a una frecuencia angular de $10 \mathrm{rad} / \mathrm{s}$, en el modo controlado (10 \%) de acuerdo con las recomendaciones del NCHRP 459 (Bahia et al. 2001). La Figura 2 muestra un ejemplo de los resultados del ensayo donde el Módulo complejo $G^{*}$ a cortante tiende a disminuir con el paso del tiempo. A cierto punto se presenta una caída drástica en el módulo que coincide con el ángulo de fase máximo encontrado durante el ensayo. Este punto se considera como el inicio del estado de falla por fatiga del material. Este ensayo se recomienda aplicar en el ligante virgen, y en la proporción o combinación RAP/ligante virgen seleccionada para el diseño de mezcla, con el objetivo de evaluar el potencial aumento en la susceptibilidad al agrietamiento por fatiga aportado por el ligante envejecido. 

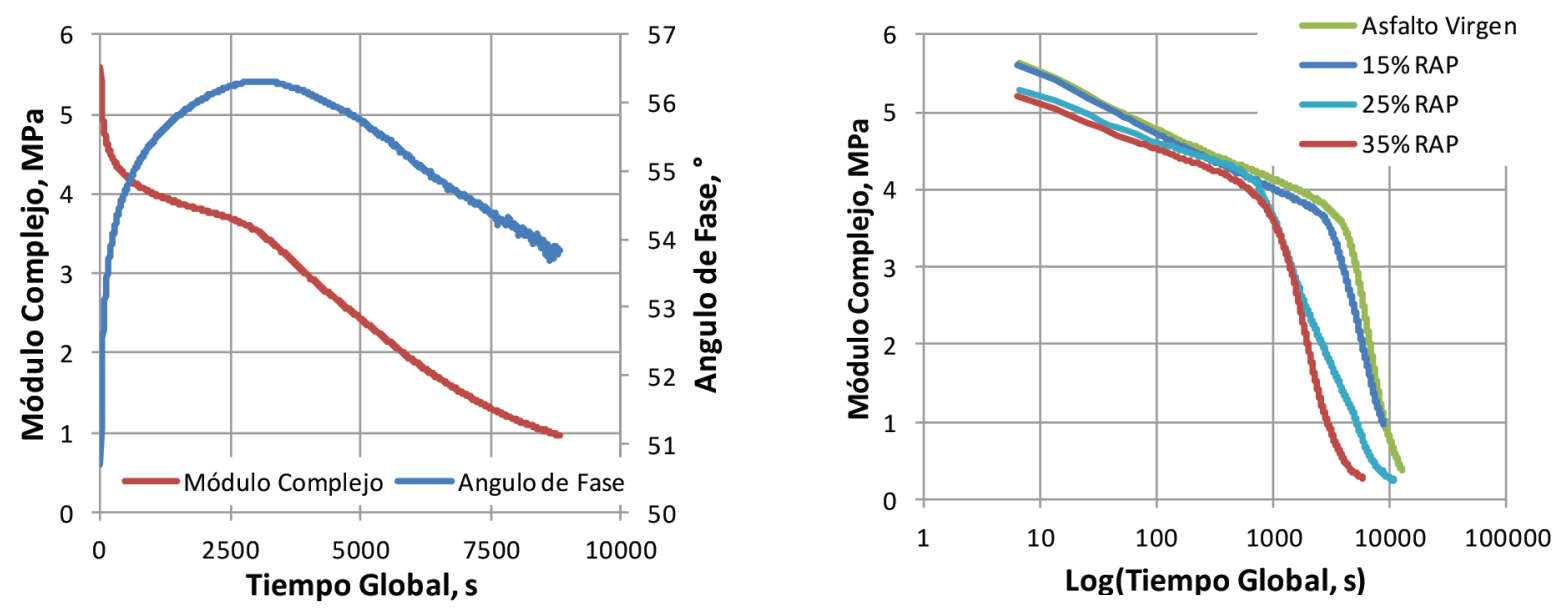

Figura 2. Ejemplo de ensayo de fatiga.

\section{Ensayo para evaluar la susceptibilidad al daño por humedad}

Debido a las condiciones ambientales y la naturaleza de los materiales de zonas tropicales, el potencial del daño por la presencia de humedad es de especial interés para las mezclas de Costa Rica y otros países con alta precipitación. Para caracterizar la susceptibilidad a la humedad del ligante, se recomienda una prueba basada en una modificación realizada al equipo llamado PATTI (por sus siglas en inglés) que mide la fuerza de tracción requerida para despegar el ligante de la superficie de agregado (BBS, en inglés) (Morales et al. 2011). Este tipo de análisis es muy útil en la identificación del tipo de falla que es probable que ocurra por adherencia entre el agregado y el ligante asfáltico o debido a la fuerza de cohesión o la durabilidad del ligante asfáltico o ambas. La Figura 3 muestra el equipo recomendado y un ejemplo de los resultados de las pruebas de tracción (Aguiar-Moya et al. 2013). El ensayo trata, básicamente, de la medida de la resistencia a la tracción (POTS) y se realiza bajo dos tipos de condicionamiento, 24 horas en estado seco y 96 horas en estado húmedo. Además, la pérdida porcentual de resistencia a la tracción entre las condiciones seca y húmeda se puede utilizar como medida de la susceptibilidad a la humedad. Este ensayo se recomienda aplicar en el ligante virgen, y en la proporción o combinación RAP/ligante virgen seleccionada para el diseño de mezcla, con el objetivo de evaluar el potencial aumento en resistencia o daño por humedad aportada por el ligante envejecido.

\section{Ensayo de deformación permanente}

El ensayo Multi Stress Creep Recovery (MSCR), definido en ASTM D 7405, fue introducido recientemente para evaluar los ligantes asfálticos a temperaturas de servicio elevadas, en particular para evaluar el esfuerzo o carga de resistencia. Esta prueba permite la clasificación de ligantes en la alta temperatura PG, de acuerdo con la carga de tráfico, a partir de la norma, a la carga de tráfico pesado, muy pesado y extremo. Este ensayo se recomienda aplicar en el ligante virgen, y en la proporción o combinación RAP/ligante virgen seleccionada para el diseño de mezcla con el objetivo de evaluar el potencial aumento en resistencia a la deformación permanente aportada por el ligante envejecido.
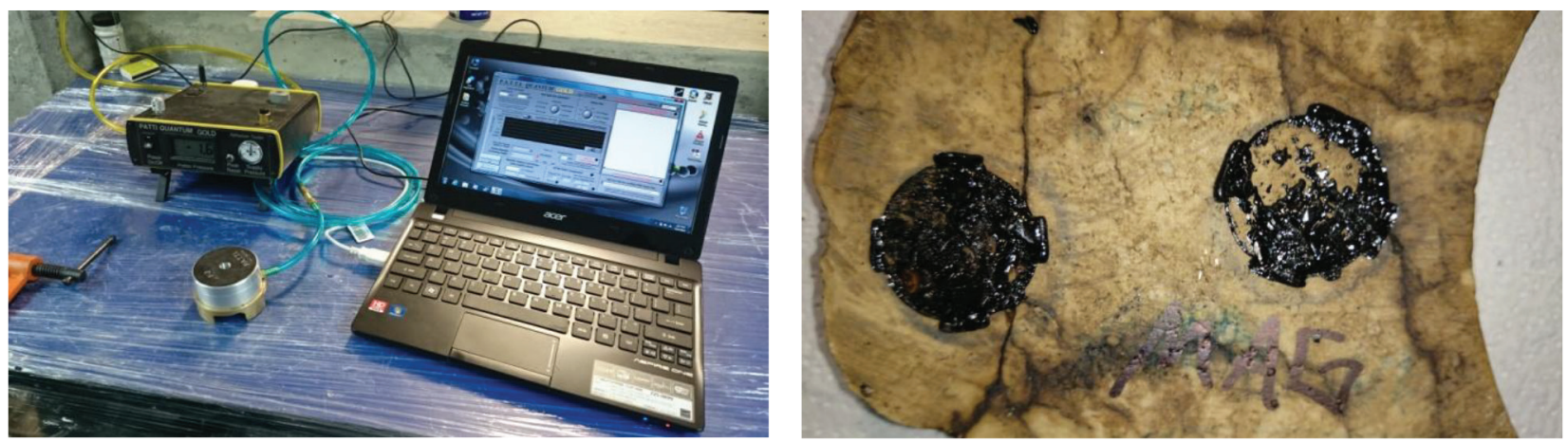

Figura 3. Ejemplo de equipo PATTI y resultados del ensayo. 


\section{Pruebas adicionales para evaluar las propiedades del ligante asfáltico}

\section{Análisis Termogravimétrico}

El análisis termogravimétrico (TGA) es un método de análisis térmico en la que los cambios en propiedades físicas y químicas de los materiales se mide como una función del aumento o disminución de los ciclos de temperatura (con velocidad de calentamiento constante), o como una función del tiempo (con temperatura constante y/o pérdida constante de masas) (Coats y Redgern, 1963; Leiva-Villacorta et al. 2014). TGA se utiliza comúnmente para determinar características seleccionadas de materiales que exhiben ya sea la pérdida de masa o ganancia debido a la descomposición, oxidación, o la pérdida de volátiles del aglutinante de asfalto. La Figura 4 muestra un ejemplo de las pruebas realizadas en ligantes envejecidos y sin envejecer. Como se muestra, la pérdida de masa del ligante virgen (no envejecido) es mayor que el ligante envejecido a lo largo del incremento de temperatura. A una temperatura de mezcla típica de $160{ }^{\circ} \mathrm{C}$ hay una diferencia significativa en la pérdida de peso calculada entre muestras. Además, una temperatura más baja de descomposición (comienzo de la aceleración del ritmo de pérdida de peso) se puede observar para el ligante no envejecido.

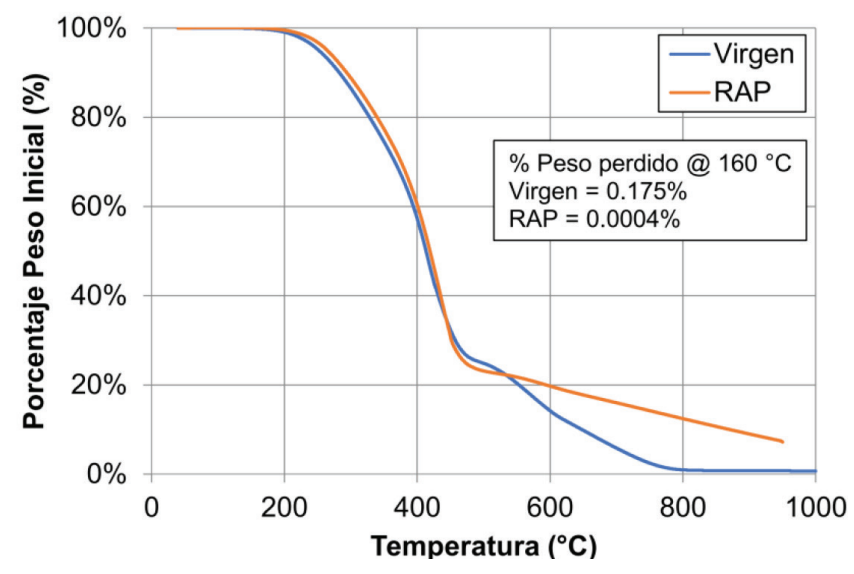

Figura 4. Ejemplo de análisis termogravimétrico.

\section{Análisis de Calorimetría de Barrido Diferencial}

El análisis de calorimetría de barrido diferencial (DSC) se utiliza ampliamente para la determinación de las transiciones térmicas provocadas por las transiciones de primer orden, tales como la fusión y la cristalización de las especies cristalizables (LeivaVillacorta et al. 2014; Elseifi et al. 2010). La transición vítrea, Tg, acreditado como un fenómeno de segundo orden que tiene lugar en la región amorfa de la muestra, puede ser también definido por DSC, pero depende en gran medida de la naturaleza del material y su contenido de fracciones cristalizables (Bahia y Anderson, 1993). Por debajo de la temperatura de transición vítrea, el asfalto se comporta como un cristal y aparece frágil y afecta al comportamiento a la fatiga del ligante y la mezcla. La Figura 5 muestra un ejemplo de la prueba DSC realizada en ligantes no envejecidos y envejecidos. Esta figura muestra que una mayor cantidad de calor está siendo introducido en el sistema, de la muestra envejecida, con el fin de producir un proceso endotérmico tal como fusión. Esta cantidad de energía adicional se ve reflejada durante la producción de mezclas asfálticas con material de RAP. A mayor contenido de RAP no sólo se tiende a elevar la temperatura del agregado virgen para minimizar la cantidad de humedad que contiene el RAP, sino también para que el asfalto envejecido se comporte de manera fluida durante el procedo de mezclado. También se espera obtener temperaturas más altas de transición vítrea ( $\mathrm{Tg}$ ) y mayor porcentaje de fracciones a cristalizar durante la muestra envejecida que puede afectar negativamente a su comportamiento a la fatiga.

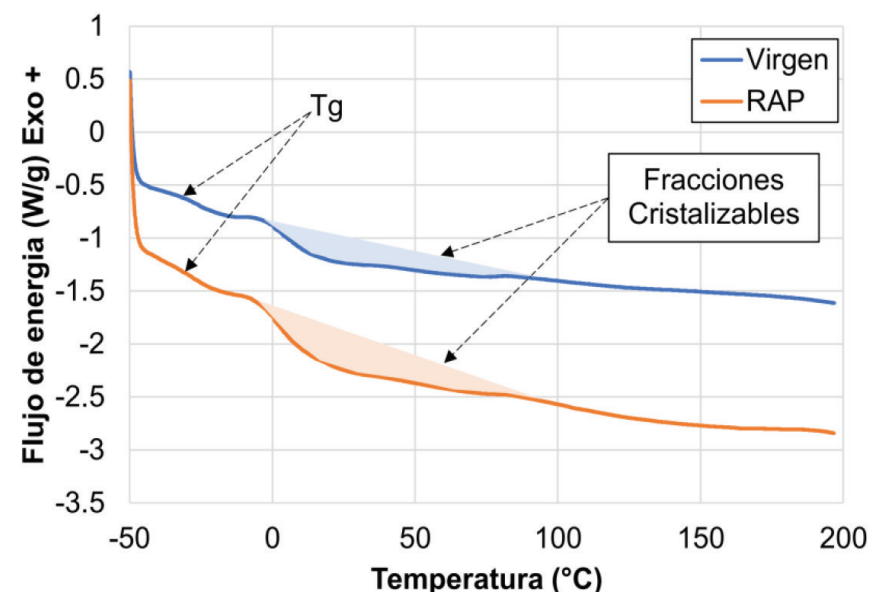

Figura 5. Ejemplo de Análisis de Calorimetría de Barrido Diferencial.

\section{Análisis Infrarrojo con Espectroscopía Infrarroja de Transformada de Fourier}

Este análisis de infrarrojo del espectro infrarrojo (FTIR) es una técnica utilizada para identificar y cuantificar cantidades de materiales conocidos y desconocidos (Leiva-Villacorta et al. 2014; Thermo Nicolet Corporation, 2001). En esta técnica, la radiación infrarroja se hace pasar a través de una muestra; parte de esta radiación es absorbida por la muestra y parte de ella se hace pasar a través de la muestra (transmisión). El espectro resultante representa la absorción molecular y de la transmisión, la creación de una huella digital molecular de la muestra (Thermo Nicolet Corporation, 2001; Kuptsov, 1994; Mason et al. 2001). El ligante asfáltico presenta cambios físicos y químicos cuando se somete a un proceso de oxidación térmica. Esto puede ser causado por 
la pérdida de volátiles o muestras de bajo peso molecular, o incluso por la formación de enlaces de hidrógeno. Los grupos forman típicamente aglutinantes de asfalto con el envejecimiento son los ácidos carboxílicos $(-\mathrm{COOH})$, cetonas (C-CO-C), sulfóxidos (R-SO-R) y anhídridos (C-O-C) (Norin y Strömvall, 2004). Estos productos de oxidación forman grupos polares con fuerte interacción que aumenta la viscosidad y el cambio de las propiedades de flujo. En la Figura 6 se muestra un ejemplo de aumento en la intensidad y la aparición de diferentes picos entre las bandas de 800 a 1200 cm-1 (sulfóxidos) y entre las bandas 1600 a 1800 cm-1 (carboxílicos) debido a la oxidación del ligante y en este caso se observa que el grupo predomínate fue el de sulfóxidos.

Una técnica complementaria a los análisis previos es el análisis FTIR de gases, mediante el acoplado al TGA. Esta técnica permite identificar químicamente espectros infrarrojos de los gases emanados por el TGA en toda la cinética termogravimétrica del material, brindando información de los gases que se podrían emanar durante el proceso de modificación o de producción en planta.



Figura 6. Ejemplo de Análisis FTIR

\section{Análisis de contaminantes en el RAP}

Hay preocupaciones ambientales relacionadas con la posible descarga de contaminantes a partir de material fresado. Naftaleno, hidroxitoluenobutilado (BHT) y ftalato de dibutilo (DBP) son algunos de los semi-volátiles más dominantes presentes en el RAP (Norin y Strömvall, 2004; Legret et al. 2005). El material del escape de los vehículos, los neumáticos de caucho y el material de asfalto en sí son todas fuentes de emisión probables, determinados a partir de los contaminantes orgánicos liberados en los apilamientos de RAP. La técnica más utilizada para el análisis de este tipo de contaminantes es la cromatografía de gases, aunque existe una tendencia clara a favor de la cromatografía líquida (Campíns-Falcó et al. 2010, Cháfer-Pericás et al. 2008), dado los problemas de blanco que se observa o que presenta la cromatografía de gases para estos compuestos. La cromatografía líquida (CL) está ganando popularidad debido a que muchos de los contaminantes ambientales son polares, presentan baja volatilidad y son lábiles.

\section{Ensayos para evaluar desempeño de la mezcla}

Hay un número importante de ensayos bien conocidos que han sido utilizados como métodos para evaluar los diferentes tipos de deterioros en pavimentos asfálticos. Sin embargo, la mayoría de estas pruebas requieren un mayor desarrollo, estudios de rigurosidad y de precisión, y la validación de campo antes de que sean adecuados para su uso en las especificaciones.

\section{Agrietamiento por fatiga}

La información sobre el desempeño del pavimento a largo plazo, aunque escasa, sugiere que mezclas con RAP pueden ser más susceptibles a agrietamiento por fatiga. Por lo tanto, la identificación de un ensayo de fatiga simple para evaluar la calidad de una mezcla RAP es esencial. Existen varios ensayos para evaluar el comportamiento a fatiga de las mezclas asfálticas, tales como fatiga a flexotracción y ensayo Overlay. El ensayo Overlay fue desarrollado por el Instituto de Transporte de Texas y ha sido utilizado con éxito como una simple y rápida prueba de desempeño de agrietamiento por fatiga. El ensayo Overlay (TEX-248-F) se lleva a cabo en muestras recortadas de mezcla compactada en el compactador giratorio o núcleos de campo que se adhieren a una placa metálica con una separación en el centro. Las muestras de ensayo se someten a muy altos esfuerzos de tracción repetidos que dan lugar a la iniciación de grietas y la propagación hasta alcanzar la falla.

El ensayo de fatiga a flexotracción (AASHTO T 321) ha sido ampliamente utilizado en mezclas asfálticas y específicamente ha sido utilizado por varios investigadores (Shen y Carpenter, 1929; Shu et al. 2007; Monismith et al. 1961; Vargas-Nordcbeck et al. 2008) para evaluar la resistencia a la fatiga de las mezclas con RAP. Se recomienda el uso de este ensayo para diseño y verificación de la mezcla asfáltica con RAP y como complemento de control de calidad se recomienda el ensayo Overlay.

\section{Ensayo para evaluar la susceptibilidad al daño por humedad}

La susceptibilidad a la humedad de todas las mezclas normalmente se evalúa mediante la determinación de la resistencia a la tracción diametral en muestras secas y húmedas de acuerdo con AASHTO T 283. En esta prueba, presiones internas de agua en las mezclas se producen por saturación al vacío seguido de un acondicionado en agua caliente. Otra forma de medir dicha 
susceptibilidad es utilizando el dispositivo rueda cargada de Hamburgo. La prueba se lleva a cabo de acuerdo con AASHTO $\mathrm{T} 324$ y las muestras se someten a una carga estandarizada mientras están sumergidas bajo agua caliente. En este proceso, el punto de inflexión o desnudamiento del agregado se utiliza normalmente para evaluar el daño por humedad. Se recomienda el uso de este ensayo para diseño y verificación de la mezcla asfáltica con RAP y como complemento de control de calidad se recomienda AASHTO T 283.

\section{Ensayo de deformación permanente}

Diversos estudios que han utilizado ensayos mecánicos o fundamentales para evaluar las mezclas que contienen RAP han concluido que la resistencia a la deformación aumenta (Mohammad et al. 2006; Drescher et al. 1993). Dos de los ensayos que se utilizan comúnmente para evaluar la deformación permanente son AASHTO T 340 (Analizador de Pavimento Asfáltico) y AASHTO T 324 (dispositivo de rueda de Hamburgo).

\section{Ejemplo de especificaciones basadas en el desempeño de la mezcla}

Las especificaciones actuales incluidas en el Manual de especificaciones para la construcción de la Carreteras de Costa Rica CR-2010 (MOPT, 2010) incluye criterios que son aplicables para cualquier tipo de mezcla asfáltica. El Cuadro 1 muestra un ejemplo de especificaciones basadas en el desempeño de mezclas asfálticas en Costa Rica. No se espera ningún cambio de estas especificaciones que deben aplicarse en mezclas de RAP.

Cuadro 1. Ejemplo de especificaciones basadas en el desempeño de la mezcla (LanammeUCR 2013)

\begin{tabular}{|c|c|c|}
\hline Parámetro & Especificación & $\begin{array}{c}\text { Método } \\
\text { Estándar }\end{array}$ \\
\hline TSR & $\geq 80 \%$ & AASHTO T 283 \\
\hline Resistencia a la tension (secas) & $\geq 700 \mathrm{kPa}$ & AASHTO T 283 \\
\hline $\begin{array}{c}\text { Deformación Permanente en } \\
\text { equipo APA a } 60^{\circ} \mathrm{C}\end{array}$ & $\leq 2,5 \mathrm{~mm}$ & AASHTO T 340 \\
\hline $\begin{array}{c}\text { Repeticiones a la falla por fatiga } \\
\text { a } 20^{\circ} \mathrm{C} \text { para los siguientes niveles } \\
\text { de deformación: }\end{array}$ & $\geq 300000$ & AASHTO T 321 \\
$400 \mathrm{E}-6 \mathrm{~mm} / \mathrm{mm}$ & $\geq 25000$ & \\
$600 \mathrm{E}-6 \mathrm{~mm} / \mathrm{mm}$ &
\end{tabular}

\section{SIINTESIS}

En este documento se presentan técnicas adecuadas para la obtención, el almacenamiento, el procesamiento del RAP para producir mezclas de buena calidad. El pavimento de asfalto reciclado debe ser procesado adecuadamente para reducir la variabilidad en la granulometría y el contenido de asfalto. Las muestras deben ser tomadas de forma aleatoria del apilamiento de RAP procesado para identificar la variabilidad de las propiedades del material de RAP. En el proceso de reciclado de pavimentos de asfalto se recomienda que el tamaño máximo de los agregados debe ser de $38 \mathrm{~mm}$. El proceso de producción de RAP puede afectar el tamaño de las partículas y dar lugar a demasiadas partículas de gran tamaño que tendrían que ser tamizados o eliminados.

El grado de desempeño PG del asfalto debe ser ajustado para tener en cuenta la contribución del material de asfalto reciclado. Para contenidos de menos de $15 \%$ RAP no es necesario ajustar el grado PG del ligante virgen. Para contenidos de RAP entre 15 \% y $25 \%$, se recomienda reducir en un grado el PG del ligante virgen. Para contenidos de RAP mayores $25 \%$ el grado apropiado del asfalto virgen debe ser obtenido mediante los gráficos de mezcla.

Se recomienda la evaluación del desempeño de mezclas asfálticas diseñada con RAP, especialmente de alto contenido de RAP. Se recomienda la caracterización del ligante extraído del RAP y del ligante resultante de la combinación virgen/RAP por medio de pruebas reológicas, térmicas, y químicas para una mejor comprensión del ligante y el desempeño de la mezcla.

Actualmente, las especificaciones para construcción de carreteras en Costa Rica incluyen especificaciones que están basadas en el desempeño de las mezclas asfálticas convencionales. El cumplimiento de tales especificaciones debe aplicarse también a las mezclas asfálticas con RAP. 


\section{REFERENCIAS BIBLIOGRÁFICAS}

1. Aguiar-Moya, José Pablo, Loria-Salazar, Luis, Salazar, Jorge, Corrales-Azofeifa, Jose, Villegas, Ernesto, Corrales-Azofeifa, José P, Hajj, Elie Y. Evaluation of Adhesion Properties of Costa Rican Asphalt Mixtures using the Bitumen Bond Strength (BBS) and Contact Angle Measurement Tests. Transportation Research Board Annual Meeting 2013 Paper \#13-0743, Washington DC.

2. Bahia, H. U. and D. A. Anderson. Glass Transition Behavior and Physical Hardening of Asphalt Binders. Journal of the Association of Asphalt Paving Technologists, Vol. 62, 93-129. 1993.

3. Bahia, H.U., Hanson, D.I., Zeng, M., Zhai, H., Khatri, M.A., Anderson, R.M., Characterization of Modified Asphalt Binders in Superpave Mix Design, NCHRP Report No. 459, 2001, NCHRP.

4. C. Cháfer - Pericás, P. Campíns-Falcó y M.C. Prieto-Blanco, Automatic in-tube SPME and fast liquid chromatography. A 610, 268-272 (2008) Holanda.

5. Chesner, W.H., R.J. Collins and M.H. MacKay. "User Guidelines for Waste and Byproduct Materials in Pavement Construction". Publication No. FHWARD-97-148, Federal Highway Administration, McLean, VA, 1998.

6. Coats, A. W.; Redfern, J. P., Thermogravimetric Analysis: A Review. Analyst 88: 906-924. 1963.

7. Copeland, A. Reclaimed Asphalt Pavement in Asphalt Mixtures: State of the Practice. Report No. FHWA-HRT-11-021, Federal Highway Administration, McLean, Virginia, 2011.

8. Drescher, A., Kim, J. R., and D. E Newcomb, Permanent Deformation in Asphalt Concrete, Journal of Materials in Civil Engineering, Vol. 5, Issue 1, 1993 p. 112-128.

9. Elseifi, M., Mohammad, L.N., Glover, I., Negulescu, I., Daly, W.H., and Abadie, C.,Relationship between Molecular Compositions and Rheological Properties of Neat Asphalt Binder at Low and Intermediate Temperatures, Journal of Materials in Civil Engineering, 2010.

10. F. Zhou, G. Das, T. Scullion, and S. Hu. RAP Stockpile Management and Processing in Texas: State of the Practice and Proposed Guidelines. Report 0-6092-1, Texas Transportation Institute, College Station, Texas, 2010.

11. J. F. Masson, L. Pelletier, P. Collins, Rapid FTIR method for quantification of styrene-butadiene type copolymers in bitumen. Journal of Applied Polymer Science 79, $1034-1041.2001$.

12. Kandhal, P. and Mallick, R.B. Pavement Recycling Guidelines for State and Local Governments-Participant's Reference Book, Report No. FHWASA-98-042, Federal Highway Administration, Washington, DC, 1997.

13. Kuptsov, A.H. Applications of Fourier Transform Raman Spectroscopy in Forensic Science. Journal of Forensic Sciences, JFSCA, Vol. 39, No. 2, pp. 305318. 1994.

14. Legret M1, Odie L, Demare D, Jullien A., Leaching of heavy metals and polycyclic aromatic hydrocarbons from reclaimed asphalt pavement. Water Res. 2005 Sep;39(15):3675-85.

15. Leiva-Villacorta, Fabricio, Villegas-Villegas, Rafael, Aguiar-Moya, José, Salazar-Delgado, Jorge, Loría-Salazar, Luis; Effect of Aging on Rheological, Chemical, and Thermodynamic Properties of Asphalt Components. Transportation Research Board Annual Meeting 2014 Paper \#14-4668, Washington DC.

16. LM-PI-UMP-016-R1. Propuesta Mezcla Asfáltica en Caliente División 400 CR-2010, LanammeUCR, Junio, 2013.

17. McDaniel, R. and T. Nantung. Designing Superpave Mixes with Locally Reclaimed Asphalt Pavement: North Central States Jointly Fund Study. TR News, No. 239, National Academies, Washington, D.C., 2005, pp. 28-30.

18. Mohammad, L., Wu, Z., Obulareddy, S., Cooper, S., and AbadieC., Permanent Deformation Analysis of Hot-Mix Asphalt Mixtures with Simple Performance Tests and 2002 Mechanistic-Empirical Pavement Design Software", Transportation Research Record, No. 1970, (2006).

19. Monismith, C., Kenneth, E, and E. Blachmen, Asphalt Behaviour in Repeated Flexure, Proceedings of the Association of Asphalt Paving Technologists, v. 30, 1961.

20. MOPT Manual de Especificaciones Generales para la Construcción de Carreteras, Caminos y Puentes. “División 400. Pavimentos Asfálticos y Tratamientos Superficiales." Designación MOPT: CR-2010. San José, Costa Rica, 2010.

21. Morales, R., Velasquez, R., and Bahia, H. U. Measuring the Effect of Moisture on Asphalt-Aggregate Bond with the Bitumen Bond Strength Test. In Transportation Research Record: Journal of Transportation Research Board, No. 2209, Transportation Research Board 1 of the National Academies, Washington, D.C., 2011.

22. National Technology Development, LLC. Quantify the Energy and Environmental Effects of Using Recycled Asphalt and Recycled Concrete for Pavement Construction. Phase I Final Report. Report No. C-08-02, NYS Department of Transportation, 2009.

23. Norin M, Strömvall AM. Leaching of organic contaminants from storage of reclaimed asphalt pavement. Environ Technol. 2004 Mar;25(3):323-40. 
24. P. Campíns-Falcó, J.Verdú-Andrés, A.Sevillano-Cabeza, R. Herráez-Hernández, C. Molins-Legua, Y. Moliner-Martinez, J. Chromatogr. A 1217 (2010) 2695-2702.

25. Shen, S., and S. Carpenter, Application of Dissipated Energy Concept in Fatigue Cracking Endurance Limit Testing, Transportation Research Record No. 1929, Washington D. C., 2005, pp. 165-178.

26. Shu, X., B. Huang, and D. Vukosavljevic, Laboratory Evaluation of Fatigue Characteristics of Recycled Asphalt Mixture, Construction and Building Materials, 2007.

27. Solaimanian, M. and M. Tahmoressi. Variability Analysis of Hot-Mix Asphalt Concrete Containing High Percentage of Reclaimed Asphalt Pavement. Journal of the Transportation Research Board, No. 1543, National Academies, Washington, D.C., 1996, pp. 89-96.

28. Thermo Nicolet Corporation. Introduction to Fourier Transform Infrared Spectroscopy. 2001.

29. Vargas-Nordcbeck, Adriana. Watson Donald E.; Moore Jason; Jared David; Wu PeterEvaluation of the Use of Reclaimed Asphalt Pavement in Stone Matrix Asphalt Mixtures. Transportation Research Record: Journal of the Transportation Research Board, Issue 2051, 2008, pp 64-70.

30. West, R., J.R: Willis and M. Marasteanu. "Improved Mix Design, Evaluation, and Materials Management Practices for Hot Mix Asphalt with High Reclaimed Asphalt Pavement Content". NCHRP Report 752, Transportation Research Board, Washington, D.C., 2013. 